\title{
THE EFFECT OF LEFT TURNS ON THE CAPACITY OF A TRAFFIC INTERSECTION*
}

\author{
BY \\ GORDON F. NEWELL** \\ Brown University
}

\begin{abstract}
A model is proposed for the traffic flow through a fixed cycle traffic signal on a narrow two lane highway. The average flows in the two opposing lanes are computed when the queues are arbitrarily long assuming that left turns are permitted and occur with fixed probabilities in the two lanes. It is found that the existence of left turns tends to favor short cycles of the light and, under certain conditions, the competition between this effect and the obvious advantages of the long cycle light gives rise to an optimal cycle time at which the average flows have a maximum value. Some simple models for multilane highways are also considered and show optimal cycle times.

It is possible to analyse in considerable detail the queuing problem for a fixed cycle traffic light on a single lane highway at which cars are not allowed to make left or right turns [1]. The corresponding problem for a highway with two lanes of opposing traffic in which cars are allowed to make left and right turns (particularly the left turns) is much more difficult because cars that wish to turn left at a typical intersection with no special facilities for left turning cars interact with cars traveling in the opposite direction. The two queues of traffic in opposing lanes are therefore not statistically independent and one must consider the behavior of two queues simultaneously.

One of the major complications in this problem arises from the fact that cars in lane 1 may turn left if there is a sufficiently large gap in the traffic of lane 2 . To analyse this feature of the traffic flow, one must study rather detailed models which specify the size of the required gap and the statistical properties of the entering traffic. It is, however, relatively easy to determine from simple models the conditions under which the queues in both lanes will grow indefinitely. This defines the maximum traffic that the intersection can carry before both lanes have simultaneously reached capacity. If the traffic of lane 2 is below capacity, the capacity of lane 1 decreases with increasing flow in lane 2 and vice versa. The flows at which both lanes reach capacity is therefore also the minimum capacity of each lane as a function of the flow in the opposing lane.

If the incoming traffic flows in both lanes equal or exceed the average rate at which cars can go through the intersection, the queues grow indefinitely and the behavior of cars at the intersection is independent of the queue lengths or the arrival times for the incoming flow. Furthermore, there are no gaps in the flow through which a car can turn left, except at the end of the green light period. The average rate at which cars can go through the intersection under such conditions therefore depends upon only a relatively few properties of the intersection.

We consider here a model intended to simulate an intersection on a narrow two lane highway on which there is no room for passing at the intersection. Our purpose is to
\end{abstract}

*Received Jan. 4, 1958.

**Alfred P. Sloan Foundation Research Fellow. 
find the flow through the intersection when capacity is reached or exceeded in both lanes. The model is described by the following properties:

1. Each car in lane 1 will turn left with probability $p$ and each car in lane 2 will turn left with probability $p^{\prime}$.

2. During any cycle of the traffic light, cars will leave the intersection on a first come first served basis only at discrete and specified times $t_{1}<t_{2}<\cdots<t_{n}$ (the same for both lanes) with the most one car leaving the intersection in each lane at each time $t_{i}$. For a less rigid interpolation of this, we could say that we observe the intersection only at discrete times $t_{1}, t_{2}, \cdots$ chosen so that $t_{i}-t_{i-1}$ represents some average time interval between cars leaving the intersection under the ideal situation in which all cars move forward. We then approximate the exact leaving times defined in some suitable way by a neighboring value of $t_{i}$, either the preceding value of $t_{i}$, the nearest $t_{i}$, or the subsequent value of $t_{j}$. It makes little difference how one actually does this.

3. Forward moving cars or right turning cars in each lane will leave at consecutive times $t_{j}$ but a car that wishes to turn left must wait until a left turning car appears in the opposing lane or until the time $t_{n}$, the end of the green cycle of the light. We neglect the possibility that a left turning car in lane 1 might be able to turn left if a car in lane 2 turns right. This we can eliminate either by saying that there are no right turning cars or that this practically never happens even if there are right turning cars. Actually one could extend the model to include such possibilities but at the expense of complicating the algebra. Although the same techniques described below could still be applied to such an extension, the capacities would depend upon two more parameters, the probabilities of right turns in the two lanes. We prefer to sacrifice generality for simplicity, in this case.

Although we expect this model to yield a qualitatively correct description of the traffic flow under the appropriate conditions, there are certain quantitative weaknesses in this model. Assumption 1 is probably correct although there does not appear to be any experimental confirmation. Assumption 2 is a reasonable approximation for forward moving cars although it might be better to make the $t_{i}$ random variables. The main inaccuracy of this assumption, however, arises in conjunction with assumption 3 where we postulate that right turning cars leave at the same rate (measured in terms of the time intervals $t_{i}-t_{i-1}$ ) as forward moving cars and left turning cars when opposed by left turning cars also leave at this same rate. In both cases, we would expect that that the time intervals should be increased somewhat for the right and left turning cars. This model will therefore tend to underestimate slightly the influence of turns. The error is expected to be relatively small, however, in most cases of interest because the main effect of the turning cars arises from the left turning cars waiting for several time intervals, a time which is probably large compared with the excess time required to execute the left turn once the opportunity arises.

The mathematical problem of calculating the flow through the intersection can be solved by first observing that a "state" of the intersection can be defined at any time $t_{i}$ by specifying the desires of the first car in each of the two queues. From this one can calculate the probabilities of various states at any subsequent times. For all practical purposes, however, the right turn states and the forward moving states are equivalent in this model and will therefore be considered as the same states. States in which both cars move forward (or right) or both turn left are also equivalent since they both lead to the result that the two cars leave the intersection immediately and new cars appear 
in both lanes for the next value of $t_{i}$. We shall consider these states collectively as state 1 . There are two other states. We denote by state 2 that state for which the car in lane 1 wishes to turn left and the car in lane 2 wishes to go forward. State 3 will then be that state for which the car in lane 1 wishes to go forward but the car in lane 2 wishes to go left. State 2 leads to the result that the car in lane 1 remains at the intersection but the car in lane 2 leaves and a new car appears in its place at the next value of $t_{i}$.

We can define a $3 \times 3$ stochastic matrix $P$ that gives the transition probabilities from any of the three states at time $t_{i}$ to any of the three states at time $t_{i+1}$. This matrix is

$$
P=\left(\begin{array}{ccc}
p p^{\prime}+(1-p)\left(1-p^{\prime}\right) & p^{\prime} & p \\
p\left(1-p^{\prime}\right) & \left(1-p^{\prime}\right) & 0 \\
(1-p) p^{\prime} & 0 & (1-p)
\end{array}\right) .
$$

The second column, for example, describes the transitions from the state with a left turning car in lane 1 and a forward moving car in lane 2. The car in lane 1 remains as a left turning car but a new car appears in lane 2. The final states are therefore states with two left turning cars (probability $p^{\prime}$ ) or a left turning car in lane 1 and a forward moving car in lane 2 (probability $1-p^{\prime}$ ). The third column describes a symmetric situation whereas column 1 gives the result of new cars appearing in both lanes.

The first column of $P$ also gives the state probabilities at time $t_{1}$ but, for uniformity of notation, it is convenient to represent the probabilities for the states at time $t_{1}$ as given by $P e_{1}$ with $e_{1}$ the vector $(1,0,0)$. The state probabilities at time $t_{i}$ are given by the three components of the vector $P^{i} e_{1}$.

For $j \leq n-1$, the three state probabilities at time $t_{j}$ also describe the probabilities that cars leave the intersection at time $t_{i}$ in both lanes, in the second lane only, and in the first lane only. They also represent the expectations for these events. The expectations of the number of times these three events occur during the first $j$ values of $t_{k}$ are given by the components of the vector

$$
\sum_{k=1}^{j} P^{k} e_{1} \quad \text { for } j \leq n .
$$

Since at time $t_{n}$, the cars in both lanes leave the intersection regardless of which way they go, the expectations of the total number of times per cycle that cars leave in both lanes, in the second lane only, and in the first lane only are given by the components of the vector

$$
N=e_{1}+\sum_{k=1}^{n-1} P^{k} e_{1}=\sum_{k=0}^{n-1} P^{k} e_{1} .
$$

The sum of the first two components of $N$ gives the average number of cars that leave in lane 2 per cycle, thus the capacity $C_{2}$ of lane 2 in cars per cycle (provided the queue of lane 1 never vanishes). Similarly, the sum of the first and third components of $N$ gives the capacity $C_{1}$ of lane 1 in cars per cycle. The determination of $C_{1}$ and $C_{2}$ is thus reduced to evaluating the vector $N$.

$N$ can be evaluated most conveniently in terms of the eigenvectors and eigenvalues of the matrix $P$. If we let $\lambda_{1}, \lambda_{2}$, and $\lambda_{3}$ be the eigenvalues of $P ; f_{1}, f_{2}$ and $f_{3}$ the right eigenvectors; and $g_{1}, g_{2}$ and $g_{3}$ the left eigenvectors defined by

$$
P f_{i}=\lambda_{i} f_{i} \text { and } g_{i} P=\lambda_{i} g_{i},
$$


then $e_{1}$ can be expanded in terms of the $f_{i}$ to give

$$
e_{1}=\sum_{k=1}^{3} f_{k}\left(g_{k}, e_{1}\right) /\left(g_{k}, f_{k}\right),
$$

in which $\left(g_{k}, e_{1}\right)$ denotes the usual real inner product of $g_{k}$ and $e_{1}$. We then find

$$
N=\sum_{k=1}^{3}\left(\sum_{i=0}^{n-1} \lambda_{k}^{i}\right) f_{k}\left(g_{k}, e_{1}\right) /\left(g_{k}, f_{k}\right) \text {. }
$$

The sum over $j$ is a geometric series, but since $P$ is a stochastic matrix, one of the eigenvalues (which we number $\lambda_{1}$ ) is 1 whereas $\left|\lambda_{2}\right|$ and $\left|\lambda_{3}\right|$ are less than 1 . The summation over $j$ gives

$$
N=n \frac{\left(g_{1}, e_{1}\right)}{\left(g_{1}, f_{1}\right)} f_{1}+\frac{\left(1-\lambda_{2}^{n}\right)\left(g_{2}, e_{1}\right)}{\left(1-\lambda_{2}\right)\left(g_{2}, f_{2}\right)} f_{2}+\frac{\left(1-\lambda_{3}^{n}\right)\left(g_{3}, e_{1}\right)}{\left(1-\lambda_{3}\right)\left(g_{3}, f_{3}\right)} f_{3} .
$$

The eigenvalues and eigenvectors of $P$ can be found explicitly as:

$$
\begin{aligned}
& \lambda_{1}=1, \quad \lambda_{2}, \lambda_{3}=(1-p)\left(1-p^{\prime}\right) \pm\left[(1-p)\left(1-p^{\prime}\right) p p^{\prime}\right]^{1 / 2} \\
& f_{i}=\left(\begin{array}{c}
1 \\
p\left(1-p^{\prime}\right)\left(\lambda_{i}+p^{\prime}-1\right)^{-1} \\
p^{\prime}(1-p)\left(\lambda_{j}+p-1\right)^{-1}
\end{array}\right), \quad g_{i}=\left(\begin{array}{c}
1 \\
p^{\prime}\left(\lambda_{i}+p^{\prime}-1\right)^{-1} \\
p\left(\lambda_{i}+p^{\prime}-1\right)^{-1}
\end{array}\right)
\end{aligned}
$$

and so

$$
\left(g_{i}, e_{1}\right) /\left(g_{i}, f_{i}\right)=\left\{1+\frac{p p^{\prime}\left(1-p^{\prime}\right)}{\left(\lambda_{i}+p^{\prime}-1\right)^{2}}+\frac{p p^{\prime}(1-p)}{\left(\lambda_{i}+p-1\right)^{2}}\right\}
$$

Substitution of Eqs. (5) and (6) into Eq. (3) gives $N$ in terms of $p, p^{\prime}$ and $n$. The resulting explicit formula is rather awkward and it is easier to analyse the results in their present form. We consider below some special limiting cases.

Case I. $p=p^{\prime}$. The formulae for $p=p^{\prime}$ are considerably simpler than the general case although in deriving the results it is somewhat easier to recalculate the eigenvalues and eigenvectors directly from $P$ rather than using Eqs. (5) and (6) in which $\lambda_{i}+p^{\prime}-1$ vanishes. The result is

$$
N=\frac{n}{(3-2 p)}\left(\begin{array}{c}
1 \\
1-p \\
1-p
\end{array}\right)+\frac{(1-p)\left[1-(1-p)^{n}(1-2 p)^{n}\right]}{p(3-2 p)^{2}}\left(\begin{array}{r}
2 \\
-1 \\
-1
\end{array}\right)
$$

From this we obtain

$$
C_{1}=C_{2}=\frac{n(2-p)}{(3-2 p)}+\frac{(1-p)\left[1-(1-p)^{n}(1-2 p)^{n}\right]}{p(3-2 p)^{2}}
$$

This formula is plotted in Fig. 1 for various values of $n$ and $p$. In most practical cases we may consider $n \gg 1$ (it is usually in the range 10 to 20 ). The important parameter in Eq. (8) for $n \gg 1$, however, is the quantity $n p$ which is nearly proportional to the total number of left turning cars per cycle. We can distinguish two cases. If $n \gg 1$ and $n p \gg 1$ (many left turning cars per cycle), then the exponential terms in Eq. (8) are negligible and 


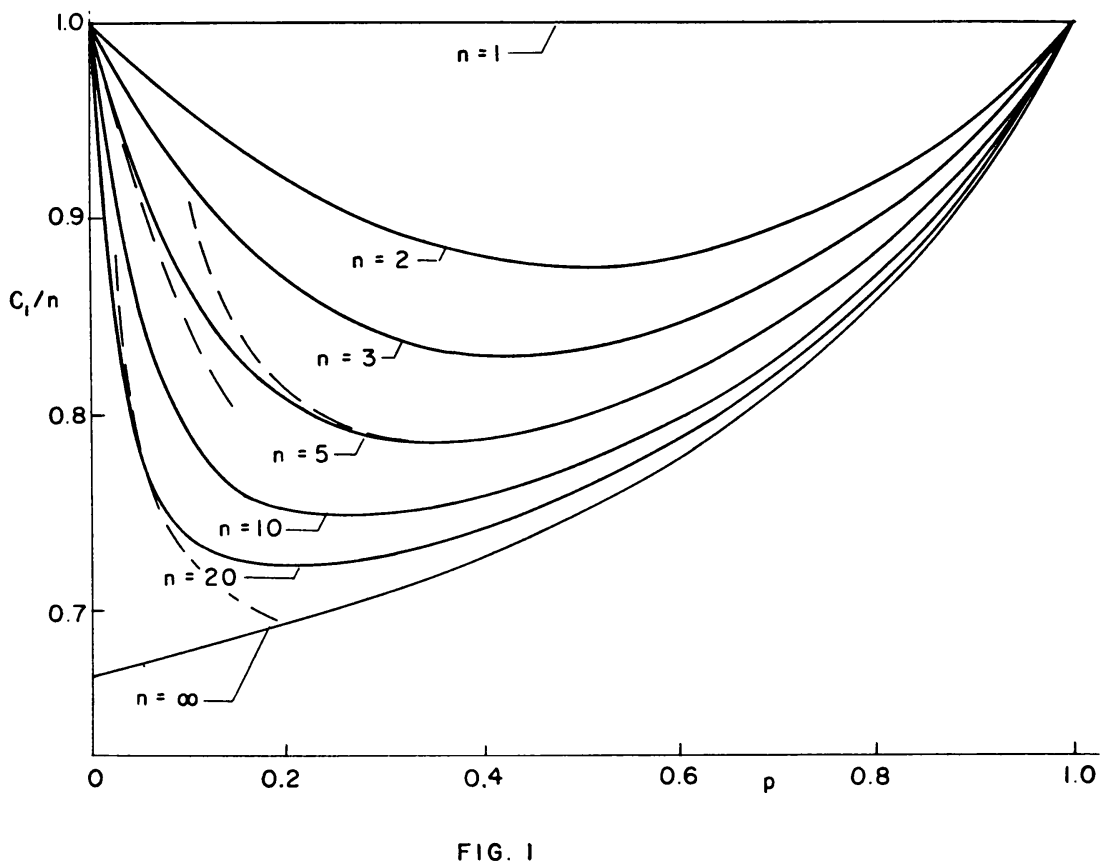

Fig. 1. The effect of left turning cars is shown by plotting $C_{1} / n$, the capacity $C_{1}$ with left turns relative to the capacity $n$ with no left turns, as a function of $n$ and the probability of left turns, $p$ (the same in the two opposing lanes). The solid curves show the exact relation given by Eq. (8) whereas the broken curves above and below the curves for $n=3$ and for $n=20$ show the limiting relations given by Eqs. (8b) and (8a), respectively. Even for $n=3$, one or the other of the limiting relations is a reasonable approximation over most of the range $0 \leq p \leq 1$.

$$
C_{1}=C_{2} \sim \frac{n(2-p)}{(3-2 p)}\left[1+\frac{(1-p)}{n p(3-2 p)(2-p)}\right],
$$

in which the second term in brackets is of order $(n p)^{-1} \ll 1$. The dominant factor is monotone increasing in $p$ which means that the more left turns the better. This curious result comes about because in this range of $p$ and $n$, the overpowering feature is the fact that the more cars there are in lane 2 that wish to turn left, the more chances there are for cars in lane 1 to turn left. In the limit $p \rightarrow 1$, all cars turn left; there is no interference between the two lanes; and $C_{1}=C_{2}=n$.

If $n \gg 1$ and $p \ll 1$, particularly if $n p$ is of order 1 or less, the exponential terms in Eq. (8) are not small but by neglecting terms of order $p$ and $n^{-1}$ as compared with 1 we find

$$
C_{1}=C_{2} \sim n\left\{\frac{2}{3}+\frac{[1-\exp (-3 n p)]}{9 n p}\right\} .
$$

An interesting feature of this formula is the fact that $C_{1} / n$, which, in a sense, represents the efficiency of the traffic flow, is a function of only $n p$. In this range, $C_{1}$ is now a monotone decreasing function of $n p$ reflecting the fact that if left turns are rare, the opportunities for left turns are also rare and the corresponding waiting period for a left turning car very long. For $n \gg 1 \gg n p$ (many cars per cycle but practically no left turns in 
one cycle), Eq. (8b) gives the proper limit $C_{1} \sim n$ but for $n \gg n p \gg 1$ (small fraction of left turns but many left turns per cycle) both Eqs. (8a) and (8b) give $C_{1} \sim 2 n / 3$.

The most interesting feature of Eq. (8), however, is the fact that $C_{1} / n$ is a monotone decreasing function of $n$, a feature which would tend to favor small values of $n$. The competition that will arise between this effect and the obvious advantages of long traffic cycles suggests that under some conditions there might be an optimal cycle time.

To investigate this possibility we note first that the traffic engineer has very little control over the values of $p$ and $p^{\prime}$ and so we consider these as fixed for any given traffic. One can, however, change $n$ by changing the length of the green period of the light. This can be accomplished by changing the ratio of the green, yellow and red periods or by changing the time of a complete cycle. Changing the ratio of red to green times tends to favor one direction of traffic at the expense of the cross traffic. We consider here only the consequences of increasing the total cycle time $T$ keeping the time of the yellow period fixed and the ratio of red to green periods fixed. Thus if $t_{y}, t_{v}$, and $t_{r}=\gamma t_{\sigma}$ represent the times of the yellow, green and red periods respectively,

$$
T=t_{\nu}+(1+\gamma) t_{\sigma},
$$

we assume that $t_{\nu}$ and $\gamma$ are kept constant while $t_{\sigma}$ and $T$ change.

We may think of $t_{o}$ as a function of $n ; t_{o}(n)$ is thus the green period that would allow $n$ forward moving cars to pass through the intersection and $T(n)$ is the corresponding cycle time under the above constraints. As a rough approximation, it is reasonable to assume that $t_{o}(n)$ is a linear function of $n$. For large $n$, after a steady flow has been reached, $t_{o}(n)$ will certainly be nearly proportional to $n$. The fact that a certain time is lost in reaching a steady flow can be roughly represented by a constant time loss. We therefore assume that

$$
t_{o}=a+b n .
$$

The values of $a$ and $b$ depend upon the units of time, but $a / b$ is dimensionless. We expect $a / b$ to be a number of order 1 .

Substitution of Eq. (10) into Eq. (8) gives

$$
T(n)=(1+\gamma) b\left\{n+\left[t_{g}+(1+\gamma) a\right] / b(1+\gamma)\right\} .
$$

By measuring $T$ in units of $(1+\gamma) b$, we can eliminate this factor so that $T$ has the form

$$
T(n)=c+n,
$$

with

$$
c=\left[t_{o}+(1+\gamma) a\right] / b(1+\gamma) .
$$

The constant $c$ must be determined experimentally but we anticipate that $c$ will be comparable with 1 and probably in the range of 1 to 3 .

The quantity of primary concern is the capacity of the intersection measured in cars per unit time, i.e. $C_{1} / T$, and we wish to know if this has a maximum with respect to the parameter $n$. One can readily show from Eq. (8) that $C_{1} / T$ is a monotone increasing function of $n$ for

$$
c \geq c_{0} \equiv \frac{(1-p)}{(2-p)(3-2 p) p}
$$


(the longer the cycle the better), whereas for $c<c_{0}, C_{1} / T$ has a maximum for some finite value of $n$. There will be a maximum for sufficiently small $p$, and for $c$ in the expected range of 1 to 3 or so, the condition on $p$ is

$$
p \lesssim 1 / 6 c \text {. }
$$

We therefore anticipate that there will be a maximum $C_{1} / T$ if $p$ is of order of about $1 / 10$ or less. It is not known to the author if this has ever been observed.

The value of $n$ at which this maximum occurs is the root of a transcendental equation and can best be found by plotting $C_{1} / T$ or its derivative with respect to $n$ vs. $n$. One can show, however, that the maximum occurs for $n p$ roughly of order 1. Figure 2 shows

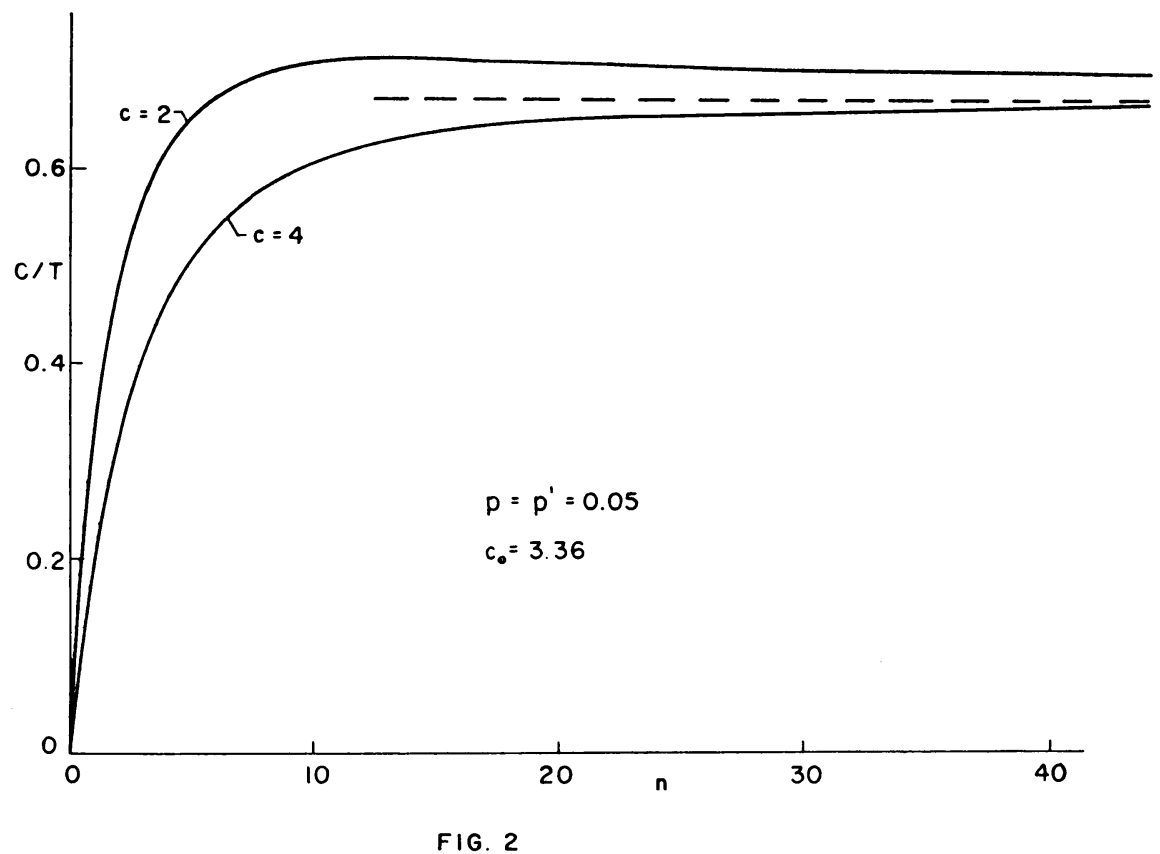

FIg. 2. The existence of a maximum capacity $C / T$ of cars per unit time as a function of $n$ is illustrated by the curve $c=2 \leq c_{0}$. The curve $c=4>c_{0}$ is monotone increasing. The curves are shown as a function of a continuous $n$ although $n$ is restricted to be integer valued.

examples of a case in which $C / T$ has a maximum and a case in which $C / T$ is monotone increasing with $n$.

Case II. $p \neq p^{\prime}, n \gg 1$. Most of the qualitative conclusions deduced for $p=p^{\prime}$ also apply for $p \neq p^{\prime}$. The exact formula for $N$ is quite complicated but for $n \gg 1$, it is possible to make approximations analogous to Eqs. (8a) and (8b). For $n \gg 1$ and either $n p \gg 1$ or $n p^{\prime} \gg 1$ or both, $\lambda_{2}^{n}$ and $\lambda_{3}^{n}$ are very small. The first term of Eq. (3) being multiplied by $n$ dominates the other two terms and gives

$$
N \sim n\left\{1+\frac{p}{p^{\prime}}\left(1-p^{\prime}\right)+\frac{p^{\prime}}{p}(1-p)\right\}\left\{\begin{array}{c}
1 \\
p\left(1-p^{\prime}\right) / p \\
p^{\prime}(1-p) / p
\end{array}\right)
$$


From this we obtain

$$
\frac{C_{1}}{n} \sim \frac{1+p^{\prime}(1-p) / p}{1+p\left(1-p^{\prime}\right) / p^{\prime}+p^{\prime}(1-p) / p}
$$

and for $C_{2}$ a corresponding expression with $p^{\prime}$ and $p$ interchanged. These reduce to the leading term of Eq. (8a) if $p=p^{\prime}$.

We note that if $p^{\prime} \rightarrow 0$ (no left turns in lane 2) but $n p \gg 1$ that $C_{1} / n \rightarrow 0$ and $C_{2} / n \rightarrow 1$. The significance of the latter is obvious; if there are no left turns in lane 2, there are no delays in lane 2 . The fact that $C_{1} / n \rightarrow 0$ arises from the fact that with probability 1 , only finitely many cars will pass the intersection in lane 1 before a left turning car appears and blocks this lane for the remainder of the cycle. Even for $n \rightarrow \infty$, we expect $C_{1}$ to remain finite and therefore $C_{1} / n \rightarrow 0$ for $n \rightarrow \infty$.

One can also see from Eq. (13) that $C_{1}$ is monotone increasing in $p^{\prime}$ for all fixed values of $p$ and monotone decreasing in $p$ for all fixed values of $p^{\prime}$. This is the type of behavior one expects since the more left turning cars in lane 2, the shorter the waiting time for a left turning car in lane 1 but the more the left turning cars in lane 1, the greater the number of delays.

The other interesting limiting case for $p \neq p^{\prime}$ is $n \gg 1, p \ll 1$ and $p^{\prime} \ll 1$. By neglecting terms of order $p^{\prime}, p$ and $n^{-1}$, we obtain

$$
\begin{gathered}
\frac{C_{1}}{n} \sim \frac{\left(1+p^{\prime} / p\right)}{\left(1+p^{\prime} / p+p / p^{\prime}\right)}+\frac{\left\{1-\exp \left[-\left(p+p^{\prime}+p^{1 / 2} p^{1 / 2}\right) n\right]\right\}\left[\left(p / p^{\prime}\right)+\left(p^{\prime} / p\right)^{1 / 2}\right]}{2 n\left(p+p^{\prime}+p^{1 / 2} p^{1 / 2}\right)\left(1+p^{\prime} / p+p / p^{\prime}\right)} \\
+\frac{\left\{1-\exp \left[-\left(p+p^{\prime}-p^{1 / 2} p^{\prime 1 / 2}\right) n\right]\right\}\left[\left(p / p^{\prime}\right)-\left(p^{\prime} / p\right)^{1 / 2}\right]}{2 n\left(p+p^{\prime}-p^{1 / 2} p^{1 / 2}\right)\left(1+p^{\prime} / p+p / p^{\prime}\right)}
\end{gathered}
$$

and for $C_{2} / n$, the corresponding expression with $p$ and $p^{\prime}$ interchanged. For $p=p^{\prime}$, this reduces to Eq. (8b).

There are several limiting cases of this that are interesting. For $n \gg n p, n p^{\prime} \gg 1$ (many left turns),

$$
\frac{C_{1}}{n} \sim \frac{1+p^{\prime} / p}{1+p^{\prime} / p+p / p^{\prime}}
$$

in agreement with the corresponding limit of Eq. (13), whereas for $n \gg 1 \gg n p, n p^{\prime}$ (no left turns), $C_{1} / n \sim 1$. For $p / p^{\prime} \ll 1$, we also find $C_{1} / n \sim 1$ but for $n p$ of order 1 and $n \gg 1 \gg n p^{\prime}$ (some left turns per cycle in lane 1 but none in lane 2) we obtain

$$
\frac{C_{1}}{n} \sim \frac{1-e^{-n p}}{n p} .
$$

This last relation obtains as a result of the fact that for $n \rightarrow \infty$ and $p \rightarrow 0$ with $n p$ finite, there is still a certain fraction of the traffic cycle that will pass traffic before a left turning car blocks the lane.

The question of existence of maximum capacities is much more complicated for $p \neq p^{\prime}$ than for $p=p^{\prime}$. Both $C_{1} / n$ and $C_{2} / n$ appear to be monotone decreasing in $n$ for all values of $p$ and $p^{\prime}$. There will certainly be a range of $p$ and $p^{\prime}$ values, with $p$ and $p^{\prime}$ sufficiently small, for which $C_{1} / T$ and $C_{2} / T$ each has a maximum as a function of $n$. For $p \neq p^{\prime}$, the values of $n$ that give the maxima of $C_{1} / T$ and $C_{2} / T$ will, however, be different. One would certainly not want to choose $n$ less than the smaller or more than 
the larger of the two values of $n$ defined by the two maxima, but any further specification of an optimal $n$ would depend upon what function of the two capacities one wishes to maximize.

Other models. The above model is neither the most difficult nor the simplest model of an intersection that one can investigate analytically. There are many relatively simple models for which one can evaluate at least the average flows when the queues are arbitrarily long. We consider below a few very simple examples.

Suppose we have a multilane highway with at least two lanes of traffic moving in each direction, no left turn light and all left turn cars restricted to the left hand lanes. As long as all the queues remain non-empty, it is impossible to make a left turn at this type of intersection except at the end of the green cycle. The behaviors of the two opposing traffics are independent of each other and can be studied separately.

If we neglect right turning cars or assume that they leave the intersection at the same rate as forward moving cars, then in analogy with the previous model, we say that there shall be $n$ cars leaving the intersection per cycle in each lane except in the one left turn lane. If a single left turning car will block the left hand lane, then $j$ cars $(j \leq n-1)$ will pass the intersection per cycle in this lane if the first $j-1$ cars in this lane are all moving forward but the $j$ th car turns left after blocking the intersection for the remainder of the cycle; $n$ cars will pass if the first $n-1$ cars move forward, independent of what the $n$th car does.

If left turning cars occur in the left hand lane with probability $p$, then the probability of $j$ cars passing the intersection in this lane is

$$
p(1-p)^{j-1} \text { for } j \leq n-1, \quad(1-p)^{n-1} \text { for } j=n .
$$

The average number of cars to leave in this lane per cycle is

$$
p \sum_{i=1}^{n-1} j(1-p)^{i-1}+n(1-p)^{n-1}=p^{-1}\left[1-\cdot(1-p)^{n}\right] .
$$

If there are $m$ lanes of traffic moving in the same direction, the total capacity of the intersection in this direction is

$$
C=(m-1) n+p^{-1}\left[1-(1-p)^{n}\right] .
$$

If, instead of having just one left turn car block the intersection, we assume that there is a space for one left turn car to wait but that a second left turn car will block the left hand lane, then the probability of $j$ cars passing the intersection per cycle in this lane is

$$
\begin{array}{ll}
(j-1) p^{2}(1-p)^{i-2} & \text { for } 2 \leq j n-1 \\
(1-p)^{n-1}+(n-1)(1-p)^{n-2} & \text { for } j=n .
\end{array}
$$

The average number of cars to leave in this lane per cycle is

$$
\begin{aligned}
p^{2} \sum_{j=2}^{n-1} j(j-1)(1-p)^{i-2}+n(1-p)^{n-1}+n(n-1) & p(1-p)^{n-2} \\
& =2 p^{-1}\left[1-(1-p)^{n}\right]-n(1-p)^{n-1} .
\end{aligned}
$$

The total capacity analogous to Eq. (16) is

$$
C=(m-1) n+2 p^{-1}\left[1-(1-p)^{n}\right]-n(1-p)^{n-1} .
$$


Each of the above situations can lead to an optimal cycle time in the sense described previously for the two lane highway. If we take $T(n)$ as in Eq. (11), then $C / T$ from Eq. (16) has a maximum as a function of $n$ if $c p(m-1)<1$ and $C / T$ from Eq. (17) has a maximum if $c p(m-1)<2$.

One could also extend these calculations for the multilane highway to include some effects of right turning cars. It would be reasonable to assume that right turn cars appear only in the right hand lanes and that the capacity of this lane is independent of what happens in other lanes. This problem can therefore be considered separately from the above.

\section{REFERENCES}

1. G. F. Newell, Statistical analysis of the flow of highway traffic through a signalized intersection, Quart. Appl. Math. 13, 353 (1956) 